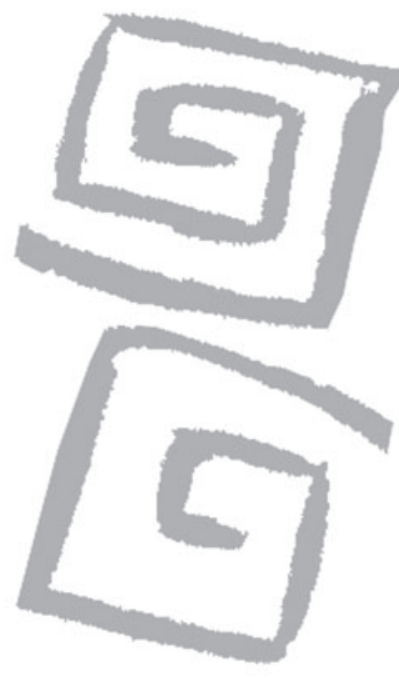

\title{
Determinantes sociodemográficos del acceso a la detección del cáncer de mama en México: una revisión de las encuestas nacionales
}

\author{
Sociodemographic determinants of access to breast \\ cancer screening in Mexico: A review of national \\ surveys
}

Agudelo Botero, Marcela ${ }^{1}$

'Administradora en Salud. Maestra en Demografía. Doctora en Estudios de Población. Investigadora en Ciencias Médicas. Centro de Información para Decisiones en Salud Pública, Instituto Nacional de Salud Pública. México.

marcela.agudelo@insp.mx
RESUMEN El objetivo de este artículo es identificar los factores que explican el acceso a la detección del cáncer de mama en México en función de las características sociodemográficas de las mujeres, de acuerdo con tres encuestas representativas en el ámbito nacional. Se realizaron estadísticas descriptivas y se emplearon técnicas de análisis de clasificación múltiple. Las variables dependientes fueron que las mujeres se hubieran realizado: 1) la autoexploración, 2) el examen clínico de mamas, o 3) la mamografía; las covariables fueron: grupos de edad, escolaridad, tipo de localidad (urbana/rural), estado conyugal, número de hijos, derechohabiencia y estrato socioeconómico. Se encontró una cobertura de detección baja y se observaron brechas entre distintos grupos femeninos según las características sociodemográficas. Por lo general, las mujeres de estratos económicos más bajos, sin derechohabiencia y con menores niveles educativos, registraron prácticas de detección inferiores a la media nacional.

PALABRAS CLAVES Cáncer de Mama; Desigualdades en la Salud; Diagnóstico Precoz del Cáncer; Demografía; México.

ABSTRACT The aim of this article is to identify factors affecting access to breast cancer screening in Mexico according to the sociodemographic characteristics of the women, using three nationally-representative surveys. Descriptive statistics were performed and multiple classification analysis techniques were used. The dependent variables were that the women had realized: 1) breast self-examination, 2) clinical breast examination, or 3) mammography; the covariates were: age group, education level, type of locality (urban/rural), marital status, number of children, enrollment in social security and socioeconomic status. A low level of screening use was detected and gaps were observed between different groups of women according to sociodemographic characteristics. In general women of lower economic strata, without enrollment in social security and with lower educational levels, showed fewer detection practices than the national average. KEY WORDS Breast Cancer; Health Inequalities; Early Detection of Cancer; Demography; Mexico. 


\section{INTRODUCCIÓN}

El cáncer de mama actualmente es un problema de salud pública que requiere ser atendido de manera prioritaria (1), dado que esta enfermedad se posiciona en el primer lugar como causa de muerte por neoplasias malignas entre la población femenina mundial $(2,3)$. Aunque su incidencia es mayor en países desarrollados, el nivel de mortalidad es más elevado en países de ingresos medios y bajos, como México (4). En este último, la tasa estandarizada de mortalidad por dicha causa ha ascendido, entre 1980 y 2009 , en un $45,3 \%$ al pasar de 11,7 a 17 muertes por cada 100 mil mujeres de 25 años en adelante (5). Para el año 2010 se estimó que la población femenina de México entre los 20 y 84 años de edad perdió en promedio 6,8 años de vida, de los cuales $2,8 \%$ se debieron a fallecimientos por cáncer de mama (6).

A pesar de los importantes avances en el conocimiento de la etiología, la prevención y el tratamiento del cáncer de mama, así como en la expansión de la cobertura de servicios de salud, en el país siguen persistiendo amplias disparidades en el acceso y atención de esta enfermedad, lo cual se ha traducido en detecciones tardías, en disminución de la sobrevida de las mujeres y, con frecuencia, en muerte (7-9). Las evidencias muestran que el control del cáncer de mama depende en gran medida de factores sociodemográficos, culturales, económicos y de la organización de los servicios de salud (10-14); sin embargo, poco se conoce sobre el peso que tienen las diferentes variables en la detección de esta dolencia.

La detección oportuna es quizá el eslabón más importante dentro de la cadena de atención, puesto que, si se hace a tiempo, existe una alta probabilidad de que las mujeres prolonguen su vida, siempre y cuando se garantice la continuidad de los procesos de intervención posteriores. Igualmente se ha planteado que determinadas características socioeconómicas de las mujeres y su ubicación geográfica las expone a mayores riesgos de morir por este tipo de cáncer, debido, entre otras cosas, a que no se detecta a tiempo (15).

Los pronósticos no son favorables y se prevé que tanto la morbilidad como la mortalidad con- tinúen incrementándose hasta tanto no se disponga de una red integral para la atención de esta neoplasia (16). De ahí que sea necesario abordar y comprender la complejidad del fenómeno para implementar medidas que contribuyan a evitar o aminorar las inequidades en salud en el territorio mexicano. En este sentido, el objetivo de este trabajo es identificar los factores que explican el acceso a la detección del cáncer de mama en México en función de las características sociodemográficas de las mujeres.

\section{MATERIAL Y MÉTODOS}

Este artículo se basa en datos de la Encuesta Nacional de Salud (ENSA) (17), la Encuesta Nacional de Salud Reproductiva (ENSAR) (18) y la Encuesta Nacional de Salud y Nutrición (ENSANUT) (19), las cuales se seleccionaron por ser representativas del ámbito nacional y por contener información sobre acceso a la detección del cáncer de mama (Cuadro 1).

En primer lugar se realizó una descripción estadística de las mujeres que realizaron prácticas de detección del cáncer de mama a través de la autoexploración, el examen clínico o la mamografía, según características sociodemográficas como: grupos de edad, escolaridad, tipo de localidad, estado conyugal, número de hijos, derechohabiencia y estrato socioeconómico (20). Dado que los rangos de edad considerados en cada encuesta difirieron entre sí, se conformaron grupos etarios que permitieron analizar la cobertura de cada técnica de detección según lo marca la Norma Oficial Mexicana NOM-041-SSA2-2011 Para la prevención, diagnóstico, tratamiento, control y vigilancia epidemiológica del cáncer de mama, donde se establece que: a) todas las mujeres deben realizarse mensualmente la autoexploración desde los 20 años de edad, b) para las mujeres mayores de 25 años de edad se recomienda el examen clínico de mamas cada año, y c) la mamografía debe practicarse en mujeres aparentemente sanas de 40 a 69 años, cada dos años. En mujeres de 70 años y más se debe realizar como parte del seguimiento de mujeres con antecedente personal de cáncer de mama y por indicación médica (21). 


\begin{tabular}{|c|c|c|c|}
\hline \multirow[t]{2}{*}{ Características } & \multicolumn{3}{|c|}{ Encuestas } \\
\hline & ENSA (2000) & ENSAR (2003) & ENSANUT (2006) \\
\hline Objetivos & $\begin{array}{l}\text { "Contribuir al conocimiento y } \\
\text { a la identificación de factores } \\
\text { genéticos, ambientales, so- } \\
\text { cioeconómicos, culturales y de } \\
\text { estilos de vida, asociados con } \\
\text { la salud y con los padecimien- } \\
\text { tos estudiados [...] Contribuir } \\
\text { en la evaluación de algunos } \\
\text { programas de salud (por ejem- } \\
\text { plo: vacunación, detección de } \\
\text { cáncer cervicouterino y otros)." }\end{array}$ & $\begin{array}{l}\text { "Obtener información acerca } \\
\text { de la salud reproductiva de las } \\
\text { mujeres mexicanas, así como } \\
\text { del conocimiento y práctica } \\
\text { de las medidas de prevención } \\
\text { y atención en la materia. Se } \\
\text { realiza con el fin de evaluar las } \\
\text { acciones realizadas y obtener } \\
\text { elementos para dar una mejor } \\
\text { orientación a los programas." }\end{array}$ & $\begin{array}{l}\text { "Estimar la frecuencia y distri- } \\
\text { bución de indicadores positivos } \\
\text { de salud, factores de riesgo de } \\
\text { enfermedad, estado nutricional } \\
\text { y deficiencias de nutrimentos, } \\
\text { enfermedades (agudas y } \\
\text { crónicas), lesiones y discapa- } \\
\text { cidad en los ámbitos nacional, } \\
\text { regional, urbano y rural, y para } \\
\text { cada una de las entidades } \\
\text { federativas en México." }\end{array}$ \\
\hline $\begin{array}{l}\text { Cobertura } \\
\text { geográfica }\end{array}$ & Nacional & $\begin{array}{l}\text { Nacional (urbano rural). } \\
\text { Estatal (urbano/rural): Chiapas, } \\
\text { Guerrero, Oaxaca, Guanajua- } \\
\text { to, Puebla, San Luis Potosí, } \\
\text { Sonora, Tamaulipas. }\end{array}$ & $\begin{array}{l}\text { Nacional y áreas rurales y } \\
\text { urbanas de cada una de las } 32 \\
\text { entidades y de todo el país. }\end{array}$ \\
\hline Diseño muestral & $\begin{array}{l}\text { Probabilístico, polietápico, } \\
\text { estratificado y por conglome- } \\
\text { rados. }\end{array}$ & $\begin{array}{l}\text { Probabilístico, polietápico y } \\
\text { estratificado. }\end{array}$ & $\begin{array}{l}\text { Probabilístico, polietápico, } \\
\text { estratificado y por conglome- } \\
\text { rados. }\end{array}$ \\
\hline $\begin{array}{l}\text { Rango de edad } \\
\text { de las mujeres de } \\
\text { la muestra }\end{array}$ & 20 años o más & 15 a 49 años & 20 años o más \\
\hline $\begin{array}{l}\text { Técnica de } \\
\text { detección }\end{array}$ & Exploración clínica de mamas & $\begin{array}{l}\text { Autoexploración } \\
\text { Exploración clínica de mamas }\end{array}$ & Mamografía \\
\hline $\begin{array}{l}\text { Total de preguntas } \\
\text { sobre cáncer de } \\
\text { mama }\end{array}$ & 7 & 17 & 12 \\
\hline Fuente: Elaboración p & a a partir de información de la ENSA ( & 7), la ENSAR (18) y la ENSANUT (19). & \\
\hline
\end{tabular}

Posteriormente se ajustaron modelos de análisis de clasificación múltiple $(22,23)$, con lo cual fue posible establecer el nivel de pronóstico de los factores incorporados y aquellos que explican en mayor medida el hecho de que las mujeres realicen prácticas de detección. En el análisis de clasificación múltiple se relacionan diversas variables predictivas con una sola variable dependiente en una tentativa de explicar la varianza de esta. Estos modelos muestran la influencia "neta" de cada una de las variables predictivas antes y después de los ajustes con otras covariables incorporadas en el análisis, con el fin de contrarrestar los efectos de las correlaciones entre variables independientes. Precisamente lo que se busca conocer es lo que queda de la relación original entre las variables independiente y dependiente, después de restar la parte que en realidad está causada por otras variables con que esta está correlacionada (23). Este análisis permite determinar la fuerza de la relación entre cada una de las variables predictivas y la variable dependiente, así como la parte de la relación que queda vigente cuando las demás variables son tomadas en cuenta (22).

Los supuestos del análisis de clasificación múltiple son similares a los usados en los modelos de regresión múltiple pero en este caso no se usa una estadística resumen de toda la variable, sino que se toman en cuenta todas las categorías de una variable predictiva, como si cada categoría fuera una variable independiente, y no correlacionada con las demás variables explicativas. Este tipo de metodología es adecuada cuando se tiene un gran número de datos, como es el caso de las encuestas poblacionales (23). 
Las variables dependientes fueron: 1) que la mujer se hubiera realizado la autoexploración de mamas, 2) que la mujer se hubiera realizado el examen clínico de mamas, o 3) que la mujer se hubiera realizado la mamografía. Como covariables estuvieron las variables sociodemográficas antes mencionadas. Los resultados de los modelos de análisis de clasificación múltiple arrojan la siguiente información:

- Media pronosticada: se refiere al efecto de cada una de las categorías (ajustadas y sin ajustar); es decir, el valor sin ajustar es el efecto neto de la categoría sin la intervención de las demás variables que se incluyen en el análisis, mientras que el índice ajustado presenta el efecto de la categoría en presencia de las otras que se contemplan en el estudio. Este valor muestra cuán afectado se ve el acceso a pruebas como la autoexploración, el examen clínico o la mamografía por determinadas características de las mujeres.

- Desviación: permite comparar la dirección del efecto de la variable (con sus categorías) respecto a la media global. Los valores de la desviación pueden ser positivos o negativos y señalan los grupos de mujeres que están por encima o por debajo del promedio nacional en el acceso a las técnicas de detección del cáncer de mama. Con este indicador se puede establecer qué mujeres tienen mayores o menores barreras para la detección de esta enfermedad.

- $\eta$ y $\beta$ : dan cuenta de la proporción explicada de cada variable, sin ajustar y ajustada, respectivamente. Ambas mediciones sirven para determinar cuál o cuáles variables sociodemográficas están explicando en mayor o menor proporción el acceso a las técnicas de detección del cáncer de mama.

Los datos se procesaron a través del Statistical Package for the Social Sciences (SPSS) en su versión 19.0

\section{RESULTADOS}

\section{Prácticas de detección del cáncer de mama}

En México se encontró una cobertura diversa de las técnicas de detección del cáncer de mama a través de la autoexploración, el examen clínico o la mamografía, lo que se corresponde con la manera en que fueron planteadas las preguntas, el período en que se levantó la información y los grupos de edad de las mujeres, los cuales fueron definidos según los objetivos de cada encuesta.

De acuerdo con la ENSAR, el 77,2\% de las entrevistadas de 20 a 49 años de edad se hicieron la autoexploración y el $47,5 \%$ de las mujeres de 25 a 49 años el examen clínico de mamas; sobre este último la ENSA reportó una cobertura de $11,2 \%$ en mujeres de 25 años o más. Finalmente, en la ENSANUT se vio que el $21,2 \%$ de las mujeres del grupo de 40-69 años se habían realizado la mamografía en los 12 meses previos al levantamiento de la encuesta. Este porcentaje se redujo al $19,2 \%$ al considerar a las mujeres de 50 años en adelante.

Según las características sociodemográficas de las mujeres, existieron distintos grados de acceso a las técnicas de detección del cáncer de mama. En la ENSA, las mujeres de áreas urbanas, con derechohabiencia, de estratos medio-alto, las no unidas, con y sin hijos, con secundaria o más y entre los 30 y los 59 años tuvieron un porcentaje de examen clínico de mamas por encima del promedio general. Las mayores diferencias, según la ENSAR, se dieron por la derechohabiencia y el estrato socioeconómico; es decir que las mujeres con derechohabiencia y de estratos socioeconómicos medio-alto tuvieron un mayor porcentaje de la autoexploración y del examen clínico de mamas frente a las que no tenían derechohabiencia y pertenecían a los estratos bajo-muy bajo. En el caso de la autoexploración, también se hallaron notables variaciones por tipo de localidad, siendo más bajo el porcentaje de utilización de la técnica entre las mujeres de áreas rurales. En cuanto a la mamografía, en la ENSANUT se evidenció que las brechas entre grupos con características sociodemográficas diversas persisten, siendo más pronunciadas dichas diferencias por derechohabiencia, nivel educativo, número de hijos y estrato socioeconómico. Del mismo modo, se observó que las mujeres entre 50 y 59 años fueron las que se realizaron relativamente más mamografías, en comparación con las mujeres de otros grupos de edad (Figura 1). 

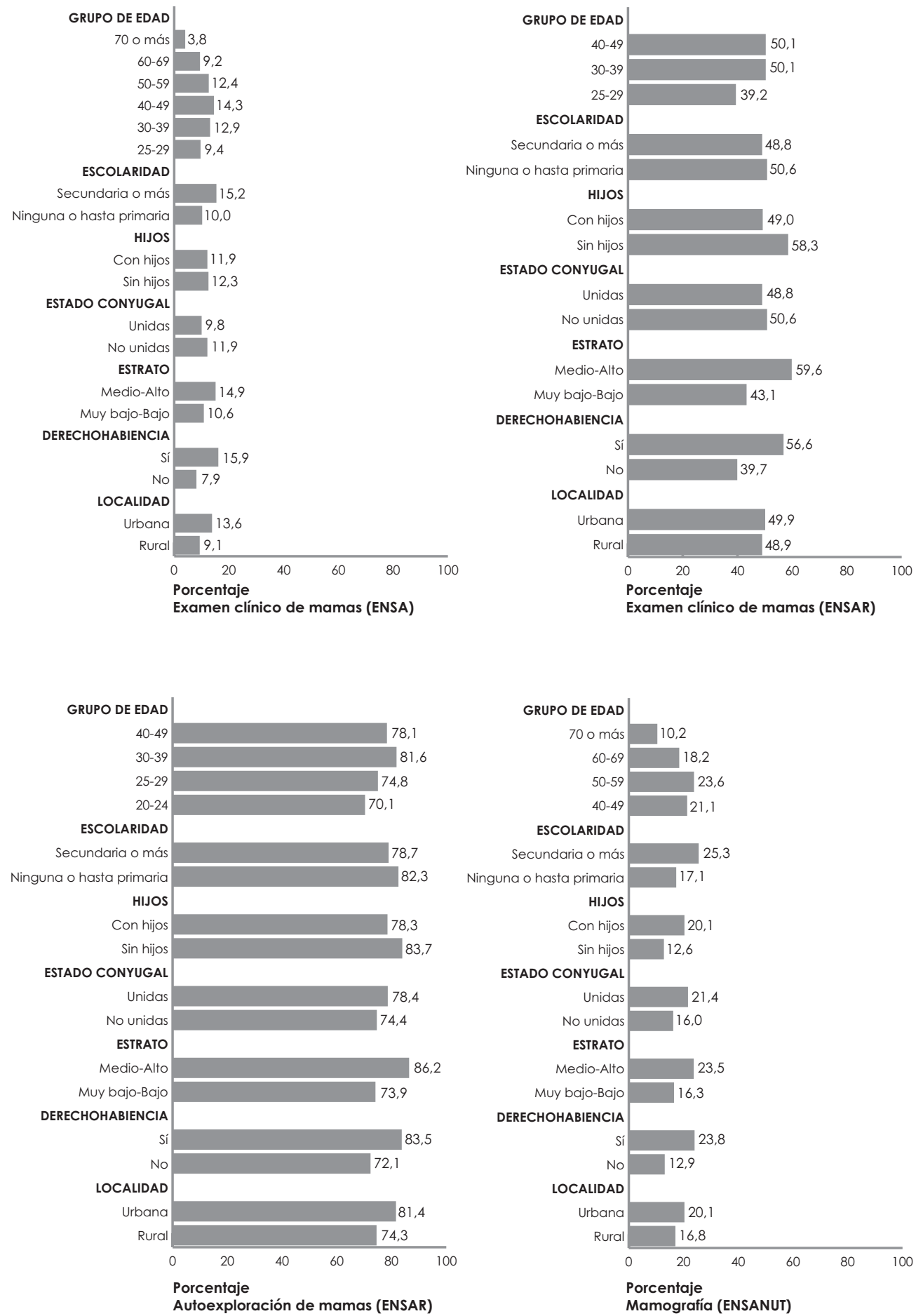

Figura 1. Distribución porcentual de las técnicas de detección de cáncer de mama utilizadas, según características sociodemográficas de las mujeres y tipo de encuestas. México, 2000, 2003 y 2006.

Fuente: Elaboración propia a partir de la ENSA (17), la ENSAR (18) y la ENSANUT (19).
ENSA = Encuesta Nacional de Salud. ENSAR = Encuesta Nacional de Salud Reproductiva. ENSANUT = Encuesta Nacional de Salud y
Nutrición.




\section{Variables asociadas con el acceso a la detección del cáncer de mama}

Según la ENSAR, las variables sociodemográficas que en su conjunto explicaron en mayor medida la autoexploración, fueron la escolaridad, el grupo de edad y el tipo de localidad. Tanto el estado conyugal como el nivel de número de hijos no fueron estadísticamente significativos en el modelo. Las mujeres de áreas urbanas, sin hijos, de estratos medio-alto, con escolaridad secundaria o más, que contaban con derechohabiencia y con edad entre los 30 y los 49 años, tuvieron medias ajustadas que superaron la media global $(0,77)$, lo que quiere decir que estas mujeres tuvieron mayor acceso a pruebas de detección del cáncer de mama mediante la autoexploración que el promedio de las mujeres entrevistadas. Efectos negativos (que se alejan de la media global) se notaron para las mujeres de zonas rurales, no unidas, de estratos muy bajo-bajo, sin escolaridad o hasta primaria, sin derechohabiencia y con 20 a 29 años de edad (Cuadro 2).

El peso de las variables respecto al examen clínico de mamas fue diferente entre la ENSA y la ENSAR. En la primera encuesta, las variables de mayor peso, teniendo en cuenta todas las variables analizadas, fueron la localidad y el estado

Cuadro 2. Resultados del análisis de clasificación múltiple de la autoexploración, según variables sociodemográficas ( $N=11.800)$. Encuesta Nacional de Salud Reproductiva (ENSAR), México, 2003.

\begin{tabular}{|c|c|c|c|c|c|c|c|c|c|}
\hline \multirow[t]{2}{*}{ Variables } & \multirow[t]{2}{*}{ Categoría } & \multirow[t]{2}{*}{$\mathrm{n}$} & \multicolumn{2}{|c|}{ Media pronosticada } & \multicolumn{2}{|c|}{ Desviación } & \multirow[t]{2}{*}{$\eta$} & \multirow[t]{2}{*}{$\boldsymbol{\beta}$} & \multirow[t]{2}{*}{ Significancia } \\
\hline & & & $\underset{\text { ajustar }}{\operatorname{Sin}}$ & Ajustada & $\underset{\text { ajustar }}{\operatorname{Sin}}$ & Ajustada & & & \\
\hline \multirow[t]{2}{*}{ Localidad } & Rural & 2.984 & 0,67 & 0,72 & $-0,11$ & $-0,05$ & \multirow{2}{*}{0,15} & \multirow{2}{*}{0,07} & \multirow{2}{*}{$* * *$} \\
\hline & Urbana & 8.816 & 0,81 & 0,79 & 0,04 & 0,02 & & & \\
\hline \multirow[t]{2}{*}{ Estado conyugal } & No unida & 1.709 & 0,77 & 0,76 & 0,00 & $-0,01$ & \multirow{2}{*}{0,00} & \multirow{2}{*}{0,01} & \multirow{2}{*}{ NS } \\
\hline & Unida & 10.091 & 0,77 & 0,77 & 0,00 & 0,00 & & & \\
\hline \multirow[t]{2}{*}{ Estratos } & $\begin{array}{l}\text { Muy bajo- } \\
\text { Bajo }\end{array}$ & 9.211 & 0,74 & 0,76 & $-0,03$ & $-0,01$ & \multirow{2}{*}{0,14} & \multirow{2}{*}{0,03} & \multirow{2}{*}{$* * *$} \\
\hline & Medio-Alto & 2.589 & 0,88 & 0,80 & 0,11 & 0,03 & & & \\
\hline \multirow[t]{2}{*}{ Escolaridad } & $\begin{array}{l}\text { Ninguna o } \\
\text { hasta primaria }\end{array}$ & 5.683 & 0,69 & 0,71 & $-0,08$ & $-0,06$ & \multirow[b]{2}{*}{0,18} & \multirow[b]{2}{*}{0,14} & \multirow[b]{2}{*}{$* * *$} \\
\hline & $\begin{array}{l}\text { Secundaria } \\
\text { o más }\end{array}$ & 6.117 & 0,85 & 0,83 & 0,07 & 0,06 & & & \\
\hline \multirow[t]{2}{*}{ Derechohabiencia } & No & 7.132 & 0,72 & 0,75 & $-0,05$ & $-0,03$ & \multirow{2}{*}{0,16} & \multirow{2}{*}{0,08} & \multirow{2}{*}{$* * *$} \\
\hline & Sí & 4.668 & 0,85 & 0,81 & 0,08 & 0,04 & & & \\
\hline \multirow[t]{4}{*}{ Grupos de edad } & $20-24$ & 1.618 & 0,69 & 0,69 & $-0,08$ & $-0,08$ & \multirow{4}{*}{0,11} & \multirow{4}{*}{0,10} & \multirow{4}{*}{$* * *$} \\
\hline & $25-29$ & 2.232 & 0,72 & 0,73 & $-0,05$ & $-0,05$ & & & \\
\hline & $30-39$ & 4.656 & 0,81 & 0,80 & 0,03 & 0,03 & & & \\
\hline & $40-49$ & 3.294 & 0,80 & 0,80 & 0,03 & 0,03 & & & \\
\hline \multirow[t]{2}{*}{ Hijos } & Sin hijo & 194 & 0,81 & 0,80 & 0,04 & 0,03 & \multirow{2}{*}{0,01} & יمת & NS \\
\hline & Con hijo & 11.606 & 0,77 & 0,77 & 0,00 & 0,00 & & 0,0 & NS \\
\hline Fuente: Elaboración pr & oia a partir de la Er & vesta $\mathrm{Nac}$ & onal de Sal & Reproductiv & (18). & & & & \\
\hline $\begin{array}{l}\eta=\text { Proporción explica } \\
* * * \\
\text { Nivel de significanci } \\
\text { NS }=\text { No significativo. }\end{array}$ & $\begin{array}{l}\text { a de cada variable } \\
(p<0,001)\end{array}$ & (sin ajustar & $\beta=$ Propo & ón explicada & le cada v & able (ajusto & & & \\
\hline
\end{tabular}


Cuadro 3. Resultados del análisis de clasificación múltiple del exámen clínico de mamas, según variables sociodemográficas (N=21.338). Encuesta Nacional de Salud (ENSA), México, 2000.

\begin{tabular}{|c|c|c|c|c|c|c|c|c|c|}
\hline \multirow[t]{2}{*}{ Variables } & \multirow[t]{2}{*}{ Categoría } & \multirow[t]{2}{*}{$\mathrm{n}$} & \multicolumn{2}{|c|}{ Media pronosticada } & \multicolumn{2}{|c|}{ Desviación } & \multirow[t]{2}{*}{$\eta$} & \multirow[t]{2}{*}{$\beta$} & \multirow[t]{2}{*}{ Significancio } \\
\hline & & & $\begin{array}{l}\text { Sin } \\
\text { ajustar }\end{array}$ & Ajustada & $\begin{array}{l}\text { Sin } \\
\text { ajustar }\end{array}$ & Ajustada & & & \\
\hline \multirow[t]{2}{*}{ Localidad } & Rural & 10.025 & 0,10 & 0,12 & $-0,02$ & $-0,01$ & \multirow{2}{*}{0,68} & \multirow{2}{*}{0,23} & \multirow{2}{*}{$* * *$} \\
\hline & Urbana & 11.363 & 0,15 & 0,13 & 0,02 & 0,01 & & & \\
\hline \multirow[t]{2}{*}{ Estado conyugal } & No unida & 17.091 & 0,13 & 0,13 & 0,01 & 0,00 & \multirow{2}{*}{0,34} & \multirow{2}{*}{0,20} & \multirow{2}{*}{$* * *$} \\
\hline & Unida & 4.297 & 0,10 & 0,11 & $-0,02$ & $-0,01$ & & & \\
\hline \multirow[t]{2}{*}{ Estratos } & $\begin{array}{l}\text { Muy bajo- } \\
\text { Bajo }\end{array}$ & 17.032 & 0,12 & 0,12 & $-0,01$ & 0,00 & \multirow{2}{*}{0,56} & \multirow{2}{*}{0,01} & \multirow{2}{*}{$* * *$} \\
\hline & Medio-Alto & 4.356 & 0,16 & 0,13 & 0,04 & 0,01 & & & \\
\hline \multirow[t]{2}{*}{ Escolaridad } & $\begin{array}{l}\text { Ninguna o } \\
\text { hasta primaria }\end{array}$ & 13.338 & 0,10 & 0,11 & $-0,02$ & $-0,01$ & \multirow{2}{*}{0,85} & \multirow{2}{*}{0,05} & \multirow{2}{*}{$* * *$} \\
\hline & $\begin{array}{l}\text { Secundaria } \\
\text { o más }\end{array}$ & 8.050 & 0,16 & 0,15 & 0,04 & 0,02 & & & \\
\hline \multirow[t]{2}{*}{ Derechohabiencia } & No & 11.550 & 0,09 & 0,09 & $-0,04$ & $-0,03$ & \multirow{2}{*}{0,12} & \multirow{2}{*}{0,10} & \multirow{2}{*}{$* * *$} \\
\hline & Sí & 9.838 & 0,17 & 0,16 & 0,04 & 0,04 & & & \\
\hline \multirow[t]{6}{*}{ Grupos de edad } & $25-29$ & 3.764 & 0,10 & 0,10 & $-0,02$ & $-0,02$ & \multirow{6}{*}{0,07} & \multirow{6}{*}{0,08} & \multirow{6}{*}{$* * *$} \\
\hline & $30-39$ & 7.227 & 0,13 & 0,13 & 0,01 & 0,01 & & & \\
\hline & $40-49$ & 5.079 & 0,15 & 0,15 & 0,02 & 0,03 & & & \\
\hline & $50-59$ & 2.709 & 0,14 & 0,14 & 0,01 & 0,01 & & & \\
\hline & $60-69$ & 1.608 & 0,11 & 0,10 & $-0,02$ & $-0,03$ & & & \\
\hline & 70 o más & 1.001 & 0,05 & 0,04 & $-0,08$ & $-0,08$ & & & \\
\hline \multirow[t]{2}{*}{ Hijos } & Sin hijo & 187 & 0,13 & 0,13 & 0,01 & 0,01 & 00 & 00 & NG \\
\hline & Con hijo & 21.201 & 0,12 & 0,12 & 0,00 & 0,00 & 0,00 & 0,00 & TNO \\
\hline Fuente: Elaboración p & pia a partir de la En & vesta $\mathrm{Nac}$ & onal de Salu & $d(17)$ & & & & & \\
\hline $\begin{array}{l}\eta=\text { Proporción explicc } \\
* * * \text { Nivel de significano } \\
\text { NS = No significativo. }\end{array}$ & $\begin{array}{l}\text { a de cada variabl } \\
(p<0,001)\end{array}$ & sin ajustar & $3=$ Propo & ón explicadc & e cada ve & iable (ajustac & & & \\
\hline
\end{tabular}

conyugal (media ajustada). En la ENSAR, la derechohabiencia y el estrato socioeconómico explicaron en mayor medida el acceso a esta prueba. En ambos modelos se encontró que a nivel individual $(\eta)$, tanto la escolaridad como el estrato socioeconómico inciden, en gran parte, en el empleo de dicha técnica de detección. Los resultados de las dos encuestas coinciden en que las mujeres de localidades urbanas, de estratos medio-alto, con nivel educativo elevado, con derechohabiencia (pública o privada) y de 30 años o más aumentan sus probabilidades de hacerse la prueba en cuestión $(p<0,001)$; asimismo, caracte- rísticas como ser de áreas rurales, pertenecer a estratos bajos, no tener escolaridad o hasta primaria, no tener seguridad social, estar en los rangos de edad 20 a 29, 60 a 69 y 70 años o más, y estar unida (solo en la ENSA) reduce las probabilidades de utilización del examen clínico como técnica de detección del cáncer de mama (Cuadro 3 y 4).

En la ENSANUT todas las variables sociodemográficas consideradas estuvieron estadísticamente asociadas con el acceso a la mamografía $(p<0,001)$, aunque las que la explicaron en mayor grado fueron la derechohabiencia y el grupo de edad (de manera individual y colectiva); también 
Cuadro 4. Resultados del análisis de clasificación múltiple del examen clínico de mamas, según variables sociodemográficas ( $N=10.182$ ). Encuesta Nacional de Salud Reproductiva (ENSAR), México, 2003.

\begin{tabular}{|c|c|c|c|c|c|c|c|c|c|}
\hline \multirow[t]{2}{*}{ Variables } & \multirow[t]{2}{*}{ Categoría } & \multirow[t]{2}{*}{$\mathrm{n}$} & \multicolumn{2}{|c|}{ Media pronosticada } & \multicolumn{2}{|c|}{ Desviación } & \multirow[t]{2}{*}{$\eta$} & \multirow[t]{2}{*}{$\beta$} & \multirow[t]{2}{*}{ Significancia } \\
\hline & & & $\begin{array}{l}\text { Sin } \\
\text { ajustar }\end{array}$ & Ajustada & $\begin{array}{l}\text { Sin } \\
\text { ajustar }\end{array}$ & Ajustada & & & \\
\hline \multirow[t]{2}{*}{ Localidad } & Rural & 2.512 & 0,37 & 0,44 & $-0,10$ & $-0,02$ & \multirow{2}{*}{0,11} & \multirow{2}{*}{0,03} & \multirow{2}{*}{$* * *$} \\
\hline & Urbana & 7.670 & 0,49 & 0,47 & 0,03 & 0,01 & & & \\
\hline \multirow[t]{2}{*}{ Estado conyugal } & No unida & 1.452 & 0,45 & 0,44 & $-0,01$ & $-0,03$ & \multirow{2}{*}{0,01} & \multirow{2}{*}{0,02} & \multirow{2}{*}{ NS } \\
\hline & Unida & 8.730 & 0,47 & 0,47 & 0,00 & 0,00 & & & \\
\hline \multirow[t]{2}{*}{ Estratos } & $\begin{array}{l}\text { Muy bajo- } \\
\text { Bajo }\end{array}$ & 7.733 & 0,42 & 0,45 & $-0,04$ & $-0,01$ & \multirow{2}{*}{0,15} & \multirow{2}{*}{0,05} & \multirow{2}{*}{$* * *$} \\
\hline & Medio-Alto & 2.449 & 0,60 & 0,51 & 0,13 & 0,05 & & & \\
\hline \multirow[t]{2}{*}{ Escolaridad } & $\begin{array}{l}\text { Ninguna o } \\
\text { hasta primaria }\end{array}$ & 5.020 & 0,38 & 0,41 & $-0,08$ & $-0,05$ & \multirow{2}{*}{0,17} & \multirow{2}{*}{0,10} & \multirow{2}{*}{$* * *$} \\
\hline & $\begin{array}{l}\text { Secundaria } \\
\text { o más }\end{array}$ & 5.162 & 0,55 & 0,51 & 0,08 & 0,05 & & & \\
\hline \multirow[t]{2}{*}{ Derechohabiencia } & No & 6.011 & 0,38 & 0,41 & $-0,08$ & $-0,06$ & \multirow{2}{*}{0,20} & \multirow{2}{*}{0,14} & \multirow{2}{*}{$* * *$} \\
\hline & Sí & 4.171 & 0,58 & 0,55 & 0,12 & 0,08 & & & \\
\hline \multirow[t]{3}{*}{ Grupos de edad } & $25-29$ & 2.232 & 0,40 & 0,41 & $-0,06$ & $-0,06$ & \multirow{3}{*}{0,07} & \multirow{3}{*}{0,06} & \multirow{3}{*}{$* * *$} \\
\hline & $30-39$ & 4.655 & 0,48 & 0,47 & 0,01 & 0,01 & & & \\
\hline & $40-49$ & 3.295 & 0,49 & 0,49 & 0,03 & 0,03 & & & \\
\hline \multirow[t]{2}{*}{ Hijos } & Sin hijo & 108 & 0,56 & 0,51 & 0,09 & 0,05 & \multirow{2}{*}{0,02} & \multirow{2}{*}{0,01} & NS \\
\hline & Con hijo & 10.074 & 0,46 & 0,46 & 0,00 & 0,00 & & & . \\
\hline Fuente: Elaboración pr & pia a partir de la Er & vesta $\mathrm{NaC}$ & onal de Sal & Reproductiv & (18). & & & & \\
\hline $\begin{array}{l}\eta=\text { Proporción explico } \\
* * * \text { Nivel de significanc } \\
\text { NS }=\text { No significativo. }\end{array}$ & $\begin{array}{l}\text { la de cada variable } \\
(p<0,001)\end{array}$ & (sin ajusta & $\beta=$ Propo & ón explicada & le cada & able lajust & & & \\
\hline
\end{tabular}

resultó importante el estrato socioeconómico. Las mujeres no unidas, sin hijos, de estratos socioeconómicos medio-alto, con escolaridad (secundaria o más), con derechohabiencia y las de 60 años de edad o más, superaron el nivel medio $(1,81)$ de cobertura mamográfica, en contraposición con las mujeres unidas, con hijos, de estratos socioeconómicos bajos, sin escolaridad o hasta primara, sin derechohabiencia y entre los 40 y 59 años (Cuadro 5).

\section{DISCUSIÓN}

México carece de un registro universal de mujeres con cáncer de mama y poco se conoce sobre los procesos de atención de esta enfermedad, desde la detección hasta su tratamiento y control $(24,25)$. No obstante, es posible explorar sobre las prácticas de detección de esta afección por medio de información captada en encuestas poblacionales. Estas tienen la ventaja de incluir tanto a las mujeres que acceden o hacen uso de los servicios de salud como a aquellas que, por diversas circunstancias, no tienen contacto con dichos servicios. En este estudio se evidenció la carencia de datos sólidos y consistentes para la medición de este fenómeno, situación que ya ha sido planteada previamente por otros autores $(1,26)$.

El tema de las prácticas de detección del cáncer de mama se ha abordado de manera marginal en las distintas encuestas del país sin que se 
tenga hasta ahora criterios homologados de recolección de datos que permitan una comparación directa de los resultados en el tiempo. Muestra de esto pueden ser los valores calculados por medio de la ENSA (2000) y la ENSAR (2003) donde se indagó sobre la exploración de mamas por parte de personal médico. Al observar la forma en que se preguntó sobre esta técnica se puede intentar explicar las variaciones en los porcentajes de cobertura obtenidos. En la primera encuesta mencionada la pregunta fue: Durante los últimos meses ¿acudió al módulo de medicina preventiva para la detección de cáncer de mama? (exploración clínica) (17), mientras que en la ENSAR fue: ¿Algún médico o personal de salud le ha revisado sus senos para ver si tiene tumores o bolitas? (18). De este modo, el diseño del cuestionario pudo influir en las respuestas de las entrevistadas, teniendo en cuenta además que cada encuesta tuvo poblaciones de referencia que variaron entre sí (ver Cuadro 1).

Pese a estas limitaciones de orden metodológico, en este artículo se presentaron las principales variables sociodemográficas asociadas con el acceso a las distintas técnicas de detección del cáncer de mama (autoexploración, examen clínico y mamografía), de acuerdo con datos de tres encuestas con dominio nacional: ENSA (2000), ENSAR (2003) y ENSANUT (2006) (17-19) donde se pusieron en evidencia las profundas desigualdades

Cuadro 5. Resultados del análisis de clasificación múltiple de la mamografía, según variables sociodemográficas (N=12.281). Encuesta Nacional de Salud y Nutrición (ENSANUT), México, 2006.

\begin{tabular}{|c|c|c|c|c|c|c|c|c|c|}
\hline \multirow[t]{2}{*}{ Variables } & \multirow[t]{2}{*}{ Categoría } & \multirow[t]{2}{*}{$\mathbf{n}$} & \multicolumn{2}{|c|}{ Media pronosticada } & \multicolumn{2}{|c|}{ Desviación } & \multirow[t]{2}{*}{$\eta$} & \multirow[t]{2}{*}{$\boldsymbol{\beta}$} & \multirow[t]{2}{*}{ Significancio } \\
\hline & & & $\begin{array}{c}\text { Sin } \\
\text { ajustar }\end{array}$ & Ajustada & $\begin{array}{c}\text { Sin } \\
\text { ajustar }\end{array}$ & Ajustada & & & \\
\hline \multirow[t]{2}{*}{ Localidad } & Rural & 3.169 & 1,80 & 1,81 & $-0,01$ & 0,00 & \multirow{2}{*}{0,04} & \multirow{2}{*}{0,01} & \multirow{2}{*}{$* * *$} \\
\hline & Urbana & 9.112 & 1,83 & 1,80 & 0,03 & 0,00 & & & \\
\hline \multirow[t]{2}{*}{ Estado conyugal } & No unida & 4.829 & 1,84 & 1,82 & 0,03 & 0,01 & \multirow{2}{*}{0,07} & \multirow{2}{*}{0,03} & \multirow{2}{*}{$* * *$} \\
\hline & Unida & 7.452 & 1,79 & 1,80 & $-0,02$ & $-0,01$ & & & \\
\hline \multirow[t]{2}{*}{ Estratos } & $\begin{array}{l}\text { Muy bajo- } \\
\text { Bajo }\end{array}$ & 7.176 & 1,77 & 1,78 & $-0,04$ & $-0,03$ & \multirow[t]{2}{*}{0,09} & \multirow[t]{2}{*}{0,06} & \multirow[t]{2}{*}{$* * *$} \\
\hline & Medio-Alto & 5.105 & 1,84 & 1,83 & 0,03 & 0,02 & & & \\
\hline \multirow[t]{2}{*}{ Escolaridad } & $\begin{array}{l}\text { Ninguna o } \\
\text { hasta primaria }\end{array}$ & 9.065 & 1,75 & 1,78 & $-0,06$ & $-0,03$ & \multirow{2}{*}{0,09} & \multirow{2}{*}{0,04} & \multirow{2}{*}{$* * *$} \\
\hline & $\begin{array}{l}\text { Secundaria } \\
\text { o más }\end{array}$ & 3.216 & 1,83 & 1,82 & 0,02 & 0,01 & & & \\
\hline \multirow[t]{2}{*}{ Derechohabiencia } & No & 5.100 & 1,76 & 1,77 & $-0,05$ & $-0,04$ & \multirow{2}{*}{0,14} & \multirow{2}{*}{0,12} & \multirow{2}{*}{$* * *$} \\
\hline & Sí & 7.181 & 1,87 & 1,86 & 0,06 & 0,05 & & & \\
\hline \multirow[t]{4}{*}{ Grupos de edad } & $40-49$ & 4.971 & 1,79 & 1,80 & $-0,02$ & $-0,01$ & & & \\
\hline & $50-59$ & 2.962 & 1,76 & 1,77 & $-0,04$ & $-0,04$ & \multirow{3}{*}{0,11} & \multirow{3}{*}{0,09} & \multirow{3}{*}{$* * *$} \\
\hline & $60-69$ & 2.173 & 1,82 & 1,81 & 0,01 & 0,01 & & & \\
\hline & 70 o más & 2.175 & 1,90 & 1,88 & 0,09 & 0,07 & & & \\
\hline \multirow[t]{2}{*}{ Hijos } & Sin hijo & 1.356 & 1,87 & 1,86 & 0,07 & 0,05 & \multirow{2}{*}{0,06} & \multirow{2}{*}{0,05} & $* * *$ \\
\hline & Con hijo & 10.925 & 1,80 & 1,80 & $-0,01$ & $-0,01$ & & & \\
\hline Fuente: Elaboración pr & bia a partir de la Er & vesta $\mathrm{Nac}$ & nal de Sa & d y Nutrición & & & & & \\
\hline
\end{tabular}


que existen en la detección de esta enfermedad, afectando especialmente a las mujeres más pobres del país (5,6,27-29). También se encontró que las coberturas de detección del cáncer de mama, a través de las distintas técnicas, son insuficientes a la luz de la NOM-041-SSA2-2011 donde se indica que las mujeres, según el grupo de edad, deben ser objeto de acciones de prevención, ya sea de manera individual o con el apoyo del personal de salud (21).

En general, se observó que determinadas condiciones sociodemográficas como no ser derechohabiente, ser de estratos socioeconómicos bajos, tener nula o baja escolaridad, pertenecer a zonas rurales, ponen en mayor desventaja a las mujeres para acceder o utilizar servicios de salud para la inspección de las mamas. Sin embargo, no se puede asegurar que solo estas mujeres son quienes encuentran barreras en la detección, ya que en lo concerniente al cáncer de mama la situación se torna compleja en todas sus dimensiones y para todos los grupos. En este sentido, se sugiere dirigir investigaciones que permitan establecer los obstáculos que cada comunidad, estado o región presenta, teniendo en cuenta las variables individuales y contextuales que los rodea.

Además de ampliarse la cobertura de detección mediante mamografía, especialmente a las mujeres de 50 a 69 años, y reforzar el empleo de la autoexploración en mujeres de 20 años o más y del examen clínico de mamas en la población femenina a partir de los 25 años, es fundamental garantizar que dichas técnicas se realicen con la calidad necesaria para lograr la detección oportuna de la enfermedad. Para ello, es imprescindible la permanente capacitación del personal de salud y la difusión de mensajes claros y estandarizados a todas las mujeres con el fin de impactar en sus actitudes y comportamientos (30,31). Al mismo tiempo deberá disponerse de una red integral de atención, que incluya recursos humanos, físicos y financieros, con la cual sea posible completar eficazmente las demás fases de diagnóstico, tratamiento y control de este padecimiento $(32,33)$.

Si bien no se ha demostrado una disminución de la mortalidad por medio de la autoexploración y el examen clínico de mamas, estas técnicas pueden coadyuvar en la detección temprana de signos y síntomas desencadenantes del cáncer de mama $(15,34)$, por lo que estas intervenciones no pueden deslindarse de un marco de respeto por la privacidad de las mujeres y de sus fundamentos ideológicos, culturales y religiosos (35). Junto con lo anterior se requiere implementar estrategias encaminadas al mejoramiento de las condiciones de vida de la población, como lo son la escolaridad formal de las mujeres, los cambios en estilos de vida nocivos para la salud y el desarrollo de infraestructura física para lograr romper las barreras de acceso y uso de servicios de salud en todos los niveles de atención.

\section{REFERENCIAS BIBLIOGRÁFICAS}

1. Knaul F, Nigenda G, Lozano R, Arreola H, Langer A, Frenk J. Cáncer de mama en México: una prioridad apremiante. Salud Pública de México. 2009;51(Supl 2):S335-S344.

2. Lozano R, Gómez H, Lewis S, Torres L, López L. Tendencias del cáncer de mama en América Latina y El Caribe. Salud Pública de México. 2009;51(Supl 2):S147-S156.

3. Porter P. Global trends in breast cancer incidence and mortality. Salud Pública de México. 2009;51(Supl 2):S141-S146.

4. Anderson B, Cazap E, Saghir N, Yip CH, Khaled M, Otero I, Adebamowo C, Harford J. Optimisation of breast cancer management in low-resource and middle-resource countries: executive summary of the Breast Health Global Initiative consensus, 2010. The Lancet Oncology. 2011;12(4):387-398.

5. De la Vara E, Suárez L, Ángeles A, Torres G, Lazcano E. Tendencias de la mortalidad por cáncer de mama en México 1980-2009. Salud Pública de México. 2011;53(5):385-393.

6. Dávila C, Agudelo M, Aguirre A. Average of years of lost life due to breast cancer and cervical cancer according to the state marginal index in Mexico, 2000 and 2010. 2013 (en prensa).

7. Palacio LS, Lazcano E, Allen B, Hernández M. Diferencias regionales en la mortalidad por cáncer de mama y cérvix en México entre 1979-2006. Salud Pública de México. 2009;51(Supl 2):S208S218. 
8. Ortega D. Diagnóstico tardío del cáncer de mama en México: Desencuentro entre las mujeres y los servicios de salud. En: Bronfman M, Denman C, compiladores. Salud reproductiva: Temas y debates. México: Instituto Nacional de Salud Pública; 2003. p. 157-180.

9. López L, Torres L, López M, Rueda C. Identificación de lesiones mamarias malignas en México. Salud Pública de México. 2001;43(3):199-202.

10. Sosa S, Walker D, Serván E. Práctica de mamografías y pruebas de papanicolaou entre mujeres de áreas rurales de México. Salud Pública de México. 2009;51(Supl 2):S236-S245.

11. Poblano O, Figueroa JE, López L. Condicionantes institucionales que influyen en la utilización del examen clínico de mama. Salud Pública de México. 2004;46(4):294-305.

12. Lazcano E, Tovar V, Alonso P, Romieu I, López L. Cáncer de mama: Un hilo conductor histórico, presente y futuro. Salud Pública de México. 1996;38(2):139-152.

13. Flores L, Salazar E, Duarte R, Torres G, Alonso $\mathrm{P}$, Lazcano $\mathrm{E}$. Factores pronósticos relacionados con la supervivencia del cáncer de mama. Salud Pública de México. 2008;50(1):119-125.

14. Anderson B, Yip H, Ramsey S, Bengoa R, Braun S, Fitch M, Groot M, Sancho-Garnier H, Tsu V. El cáncer de mama en los países con recursos limitados: Sistemas de atención de salud y políticas públicas. The Breast Journal. 2007;13(1):62-82.

15. Díaz S, Piñeros M, Sánchez O. Detección temprana del cáncer de mama: Aspectos críticos para un tamizaje organizado en Colombia. Revista Colombiana de Cancerología. 2005;9(3):93-105.

16. Martínez O, Uribe P, Hernández M. Políticas públicas para la detección del cáncer de mama. Salud Pública de México. 2009;51(Supl 2):S350-S360.

17. Secretaría de Salud, Instituto Nacional de Salud Pública. Encuesta Nacional de Salud 2000 [Internet]. México: Secretaría de Salud; 2003 [citado 11 may 2012]. Disponible en: http://ensanut.insp. $\mathrm{mx}$ /informes/ENSA_tomo1.pdf.

18. Secretaría de Salud. La salud reproductiva en México: Análisis de la Encuesta Nacional de Salud Reproductiva 2003 [Internet]. México: Centro Regional de Investigaciones Multidisciplinarias, Universidad Nacional Autónoma de México; 2007 [citado 11 may 2012]. Disponible en: http://www. crim.unam.mx/drupal/?q= node/385.
19. Instituto Nacional de Salud Pública. Encuesta Nacional de Salud y Nutrición 2006 [Internet]. México DF: Secretaría de Salud; 2006 [citado 11 may 2012]. Disponible en: http://www.insp.mx/ ensanut/ensanut2006.pdf.

20. Echarri C. Desigualdad socioeconómica y salud reproductiva: Una propuesta de estratificación social aplicable a las encuestas. En: Lerner S, Szasz I. Salud reproductiva y condiciones de vida en México. Tomo I. México: El Colegio de México; 2008.

21. Secretaría de Salud. Norma Oficial Mexicana para la prevención, diagnóstico, tratamiento, control y vigilancia epidemiológica del cáncer de mama. NOM-041-SSA2-2011. Diario Oficial de la Federación [Internet]. 9 jun 2011 [citado 11 may 2012]. Disponible en: http://dof.gob.mx/nota_detalle.php? codigo $=5194157 \&$ fecha $=09 / 06 / 2011$.

22. De Jong J. El uso del análisis de clasificación múltiple en la demografía. Santiago de Chile: CELADE; 1974.

23. Lolle $H$. Multiple Classification Analysis (MCA): An unfortunately, nearly forgotten method for doing linear regression with categorical variables. En: Symposium I Anvendt Statistik 2008. Denmark: Copenhagen Business School Press; 2008. p. 103-122.

24. Smith R, Caleffi M, Albert US, Chen T, Duffy $S$, Franceschi D, Nyström L. El cáncer de mama en los países con recursos limitados: Detección temprana y acceso a la asistencia. The Breast Journal. 2007;13(Supl 1):S16-S29.

25. López L, Suárez L, Torres S. Detección del cáncer de mama en México: síntesis de los resultados de la Encuesta Nacional de Salud. Salud Pública de México. 2009;51(Supl 2):S199-S202.

26. Agostoni C, Ríos A. Las estadísticas de salud en México: Ideas, actores e instituciones, 18102010. México: Universidad Nacional Autónoma de México; 2011.

27. Reyes H, Gómez H, Torres L, Tomé P, Galván G, González MA, Gutiérrez G. Necesidades de salud en áreas urbanas marginadas de México. Revista Panamericana de Salud Pública. 2009;25(4):328-336.

28. Aziz Z, lqbal J, Akram M. Effect of social class disparities on disease stage, quality of treatment and survival outcomes in breast cancer patients from developing countries. The Breast Journal. 2008;14(4):372-375. 
29. Martínez M, Martínez M. La mortalidad por cáncer cérvicouterino y de mama en Colombia y México como expresión de las desigualdades socioeconómicas. En: Rodríguez L. Población y salud sexual y reproductiva en América Latina. Córdoba: ALAP; 2008.

30. González LM, González MC, Nigenda G, López L. Acciones gubernamentales para la detección temprana del cáncer de mama en América Latina: Retos a futuro. Salud Pública de México. 2010;52(6):533-543.

31. González LM, González MC, Caballero M, Aguilar E. Entrenamiento de estudiantes de medicina y enfermería en cáncer de mama en México: Estudio exploratorio [Internet]. 2005 [citado 4 mar 2012]. Disponible en: http://www.tomateloapecho.org. mx/Archivos \% 20web\% 20TAP/Entrenamiento $\% 20 d e \% 20$ estudiantes $\% 20$ CAMA.pdf.
32. Eniu A, Carlson R, Aziz Z, Bines J, Hortobágyi G, Senel N, Love R, Vikram R, Kurkure A, Anderson $\mathrm{B}$. El cáncer de mama en los países con recursos limitados: Tratamiento y asignación de recursos. The Breast Journal. 2007;13(1):S43-S61.

33. Valencia A, Sánchez G, Bautista S, Torres G, Bertozzi S. Costo-efectividad de políticas para el tamizaje de cáncer de mama en México. Salud Pública de México. 2009;51(Supl 2):S296-S304.

34. Díaz EM, Cordero MA. Nódulo de mama: papel del médico general integral para su detección precoz mediante la enseñanza y el control del autoexamen de mama. Revista Cubana de Medicina General Integral. 2001;7(4):328-334.

35. Nigenda G, Caballero M, González LM. Barreras de acceso al diagnóstico temprano del cáncer de mama en el Distrito Federal y Oaxaca. Salud Pública de México. 2009;51(Supl 2):S296-S304.

\section{FORMA DE CITAR}

Agudelo Botero M. Determinantes sociodemográficos del acceso a la detección del cáncer de mama en México: una revisión de las encuestas nacionales. Salud Colectiva. 2013;9(1):79-90.

Recibido el 28 de mayo de 2012

Versión final presentada el 30 de octubre de 2012

Aprobado 12 de febrero de 2013 